\title{
Comparative outcome analysis of bleb needling of fibrotic blebs in the clinic versus the operating room: a retrospective case series
}

\author{
Vikram Ponnusamy ${ }^{1}$, Van Nguyen ${ }^{1,2}$ and Jella A. An ${ }^{1,2^{*}}$
}

\begin{abstract}
Background: To compare 6 month outcomes of bleb needling performed in the clinic vs. the operating room (OR) in adult glaucoma patients with failed bleb.

Methods: A retrospective case series of 47 eyes from 41 glaucoma patients who received needling with mitomycin $\mathrm{C}(\mathrm{MMC})$ of scarred bleb from prior bleb-forming procedures in clinic (32 eyes) vs. the OR (15 eyes), including trabeculectomy (14 eyes), ExPress shunt (16 eyes), and ab-interno XEN gel stent (17 eyes). The primary outcome was needling success, defined as IOP $\leq 18 \mathrm{mmHg}$ on 0 glaucoma medications without requiring an additional IOP lowering procedure within 6 months after needling.

Results: At 6 months, bleb needling success rate was similar when performed in the clinic vs. in the OR (28\% vs. $20 \%, P=0.54)$. Success rate was not statistically different in patients with prior trabeculectomy, ExPress shunt, and XEN gel stent ( $29 \%$ vs. $38 \%$ vs. $12 \%, P=0.26)$. When comparing clinic vs. the OR needling procedures at 6 months, there was no difference in mean IOP (14.2 vs. $14.9 \mathrm{mmHg}, P=0.73)$, mean glaucoma medications $(1.4 \mathrm{vs} .1 .7, P=$ $0.69)$, additional IOP-lowering procedure rate ( $16 \%$ vs. $27 \%, P=0.37)$, or complication rate ( $0 \%$ vs. $7 \%, P=0.32)$.

Conclusion: Bleb needling with MMC in clinic may be a safe and effective way to revise failed bleb after trabeculectomy, EXPress shunt, and XEN gel stent procedures when compared to needling in the OR.
\end{abstract}

Keywords: Trabeculectomy, ExPress shunt, XEN gel stent, Bleb needling, Bleb revision

\section{Introduction}

Glaucoma filtration surgery is a bleb-forming procedure that effectively lowers intraocular pressure (IOP). We now have a number of ways to perform bleb-forming filtration surgery. Trabeculectomy is one of the earliest and most frequently performed glaucoma surgeries worldwide since 1968 [1, 2]. ExPress shunt (Alcon Laboratories, Fort Worth, TX, USA) was found to result

\footnotetext{
* Correspondence: anja@health.missouri.edu

${ }^{1}$ University of Missouri School of Medicine, Columbia, MO, USA

${ }^{2}$ Department of Ophthalmology, Mason Eye Institute, University of Missouri Health Care, 1 Hospital Drive, Columbia, MO 65201, USA
}

in comparable but safer surgical outcomes by increasing reproducibility and preventing intraoperative anterior chamber collapse [3]. Most recently, the XEN gel stent (Allergan PLC, Irvine, CA, USA) was developed to enable bleb formation via an ab-interno approach using a collagen stent with a $45 \mu \mathrm{m}$ inner-diameter. As a result, XEN gel stent is shown to significantly decrease the risk of hypotony, duration of surgery, recovery time, and improve reproducibility of bleb formation [4]. Each of these procedures relies upon a bleb to filter out fluid from the anterior chamber to control IOP.

(c) The Author(s). 2021 Open Access This article is licensed under a Creative Commons Attribution 4.0 International License, which permits use, sharing, adaptation, distribution and reproduction in any medium or format, as long as you give appropriate credit to the original author(s) and the source, provide a link to the Creative Commons licence, and indicate if changes were made. The images or other third party material in this article are included in the article's Creative Commons licence, unless indicated otherwise in a credit line to the material. If material is not included in the article's Creative Commons licence and your intended use is not permitted by statutory regulation or exceeds the permitted use, you will need to obtain permission directly from the copyright holder. To view a copy of this licence, visit http://creativecommons.org/licenses/by/4.0/ The Creative Commons Public Domain Dedication waiver (http://creativecommons.org/publicdomain/zero/1.0/) applies to the data made available in this article, unless otherwise stated in a credit line to the data. 
Early or late fibrosis of a bleb may decrease efficacy of the filtration channel, leading to suboptimal IOP lowering and eventual failure of filtration surgery. Bleb needling with or without antifibrotic drugs is a relatively safe and effective way to remove scar tissue and improve filtration, and it can easily be done in the clinic setting on a slit lamp with few long term complications and prevent re-operation $[5,6]$. Bleb needling involves cutting through fibrotic tissue in the bleb, which reopens the channel. The concurrent use of antifibrotic medications in this procedure helps to prevent the recurrence of fibrosis by decreasing fibroblast production and scar tissue formation. However, theoretical risks of bleb needling, including bleb leaks, choroidal effusions, suprachoroidal hemorrhage, and endophthalmitis, sway many ophthalmologists to perform bleb needling exclusively in the operating room or avoid the procedure all together.

Studies evaluating success of bleb needling for trabeculectomy, ExPress shunt, and XEN gel stent show up to $40 \%$ success [7-9]. However, a number of studies evaluating the clinical outcomes after placement of a XEN gel stent report higher early postoperative bleb needling rates up to $44.1 \%$ at 1 year $[4,10-12]$. The alternative options to bleb-needling are reintroducing glaucoma medications and undergoing further laser procedures or surgeries, which may not be in the patient's best interest. Though bleb needling is a procedure that has been shown to be effective, there are no studies that compare whether bleb needling performed in clinic is as successful and as safe as bleb needling done in the operating room $(\mathrm{OR})$ in different types of bleb-forming procedures.

In this study, we compared 6 month outcomes after bleb needling procedures performed in the clinic or the OR for failed blebs after trabeculectomy, ExPress shunt, and XEN gel stent procedures in adult glaucoma patients.

\section{Materials and methods}

Institutional Review Board (IRB) approval was obtained from the University of Missouri to conduct a retrospective chart review of 47 eyes of 41 adult glaucoma patients who underwent bleb needling procedures at Mason Eye Institute between January 1, 2016 and October 31, 2018 (IRB\# 2012046). Inclusion criteria was poorly controlled IOP with or without medications and evidence of bleb fibrosis in patients with prior trabeculectomy, ExPress shunt, or XEN gel stent procedures, with minimum 6 months follow up after bleb needling. All procedures were performed by a single surgeon. All patients received 0.02-0.04 mg of mitomycin (MMC) subconjunctival injection prior to needling, and prior ExPress shunt patients received YAG laser to the inner lumen of the shunt prior to bleb needling. Exclusion criteria were any patients who received any other procedure (Kahook Dual Blade goniotomy, MicroPulse cyclophotocoagulation, Ahmed Valve placement) at the time of bleb needling. Baseline characteristics, visual acuity (VA), IOP, and number of glaucoma medications were obtained at the time of needling, at 1 week, 1 month, 2 months, and 6 months postoperatively. Location (clinic vs. the OR), additional IOP-lowering procedures required (including repeat bleb needling) within 6 months, and any adverse events were also recorded. A de-identified data set was analyzed.

The primary outcome was comparing bleb needling success in clinic vs. the OR, where success was defined as IOP $\leq 18 \mathrm{mmHg}$, on 0 glaucoma medications, and no additional IOP lowering procedure within 6 months of needling. An eye with any vision-threatening complication was considered to be failure. Secondary subgroup analyses included bleb needling success, mean IOP, mean glaucoma medications, complications, additional IOP-lowering procedures, and re-needling rate (defined as repeat needling procedures performed within 3 months of the most recent needling) between trabeculectomy, ExPress shunt, and XEN gel stent groups. Vision-threatening complications were defined as any complication leading to loss of two or more Snellen lines.

\section{Statistical analysis}

A priori power analysis with a goal power of $80 \%$ and effect size of 0.5 suggested a total goal sample size of 32 in comparing clinic vs. the OR needling. $x^{2}$-test and Fisher's exact tests were used to compare success between these subgroups. ANOVA and paired $t$-tests were used to compare percent IOP reduction and glaucoma medication reduction. Kaplan-Meier survival analysis and log-ranked test were performed to assess bleb survival. All statistical analysis and multinomial logistic modeling were performed using SPSS Statistics (IBM Corporation; Armonk, NY, USA).

\section{Ocular physical examination}

Visual acuity was measured by a technician in clinic with a patient reading a multi-letter Snellen chart from $20 \mathrm{ft}$ away with one eye at a time. IOP was measured by an ophthalmologist or a trained technician by utilizing a Goldmann applanation tonometer. The anterior segment and posterior segment exams were done by an ophthalmologist utilizing a slit lamp microscope. Patients were dilated for indirect ophthalmoscopy if there is an indication suggesting a posterior segment pathology.

\section{Bleb needling procedure}

The eye was prepped with moxifloxacin in clinic and povidone in the OR. For both locations, topical 
tetracaine drop and lidocaine gel were used for local anesthesia, and mild intravenous sedation was administered for those treated in the OR. All patients received Mitomycin-C $(0.1-0.2 \mathrm{~mL}$ of $0.2 \mathrm{mg} / \mathrm{mL})$ injected subconjunctivally with a 30 gauge needle at the site of bleb needling, massaged with a cotton-tip applicator to distribute evenly away from the limbus, and left for over $20 \mathrm{~min}$ to bind. All needling performed in clinic was done under a slit lamp microscope. A 27 or 30 gauge needle on a $1 \mathrm{~mL}$ syringe with air was used to release subconjunctival scar tissues. In failed trabeculectomies, the scleral flap was lifted with the needle and air was injected into the anterior chamber as needed to keep the anterior chamber from collapsing. In failed ExPress Shunts, one to two $8 \mathrm{~mJ}$ shots of YAG laser was directed at the internal ostium visualized by gonioscopy to clear any debris within the shunt prior to needling. In failed XEN gel stents, a 30 gauge needle was used to gently remove any subconjunctival and Tenon adhesions above and beneath the subconjunctival side of the stent; in addition, a dispersive viscoelastic was injected into the subconjunctival space as a spacer and to tamponade any heme as needed. If done in the OR, a Blumenthal conjunctival dissector was used to further release any posterior subconjunctival adhesion and the entry was sutured with 9-0 Vicryl.

\section{Results}

\section{Baseline characteristics}

The average age of patients was $71.6 \pm 8.6$ (range, 51 to 84), with females comprising $40.4 \%$ (19/47 eyes). The majority of patients were Caucasian (85.1\%). Preoperative demographic and glaucoma information is reported in Table 1.

\section{Location of needling: in clinic vs. in the OR}

Of all bleb needling procedures performed, $68.0 \%$ were in clinic and $31.9 \%$ were in the OR (Table 1). Baseline IOP was $23.3 \pm 9.1 \mathrm{mmHg}$ and $26.1 \pm 6.8 \mathrm{mmHg}$ for patients with bleb needling performed in clinic vs. the OR, respectively $(P=0.24)$. Mean baseline number of glaucoma medications was 2 classes for needling in clinic

Table 1 Baseline demographic data

\begin{tabular}{|c|c|c|c|c|}
\hline & Overall $(n=47)$ & Clinic $(n=32)$ & OR $(n=15)$ & \\
\hline Age (years), mean $\pm S D$ & $71.6 \pm 8.6$ & $73.4 \pm 7.8$ & $68.0 \pm 9.2$ & \\
\hline Female Gender, n (\%) & $19(40.4)$ & $15(46.9)$ & $4(26.7)$ & \\
\hline \multicolumn{5}{|l|}{ Ethnicity, n (\%) } \\
\hline Caucasian & $40(85.1)$ & $27(84.4)$ & $13(86.7)$ & \\
\hline Black & $6(12.8)$ & $5(15.6)$ & $1(6.7)$ & \\
\hline Asian & $1(2.1)$ & $0(0)$ & $1(6.7)$ & \\
\hline \multicolumn{5}{|l|}{ Type of Initial Procedure, n (\%) } \\
\hline Trabeculectomy & $14(29.8)$ & $6(18.8)$ & $8(53.3)$ & \\
\hline ExPress & $16(34.0)$ & $12(37.5)$ & $4(26.7)$ & \\
\hline XEN & $17(36.2)$ & $14(43.8)$ & $3(20.0)$ & \\
\hline \multicolumn{5}{|c|}{ Time Until Needling (months), mean \pm SD } \\
\hline Trabeculectomy & $77.4 \pm 48.1$ & $77.6 \pm 54.8$ & $77.2 \pm 50.5$ & $P=0.992$ \\
\hline ExPress & $39.4 \pm 24.7$ & $39.5 \pm 22.3$ & $39.2 \pm 34.1$ & $P=0.986$ \\
\hline \multirow[t]{2}{*}{ XEN } & $1.7 \pm 1.7$ & $1.8 \pm 1.9$ & $1.6 \pm 0.8$ & $P=0.877$ \\
\hline & $P<0.001^{*}$ & $P<0.001^{*}$ & $P<0.001^{*}$ & \\
\hline Baseline IOP $(\mathrm{mmHg})$, mean \pm SD & $24.2 \pm 8.5$ & $23.3 \pm 9.1$ & $26.1 \pm 6.8$ & $P=0.243$ \\
\hline Trabeculectomy & $25.1 \pm 8.4$ & $25.3 \pm 11.1$ & $24.9 \pm 6.5$ & $P=0.931$ \\
\hline ExPress & $21.6 \pm 5.1$ & $19.7 \pm 3.7$ & $27.3 \pm 4.6$ & $P=0.035^{*}$ \\
\hline \multirow[t]{2}{*}{ XEN } & $26.0 \pm 10.7$ & $25.6 \pm 10.9$ & $28.0 \pm 11.3$ & $P=0.757$ \\
\hline & $P=0.297$ & $P=0.220$ & $P=0.764$ & \\
\hline Baseline medications, mean \pm SD & $1.9 \pm 1.6$ & $2.0 \pm 1.7$ & $1.9 \pm 1.5$ & $P=0.835$ \\
\hline Trabeculectomy & $2.5 \pm 1.6$ & $3.7 \pm 0.8$ & $1.6 \pm 1.4$ & $P=0.006^{*}$ \\
\hline ExPress & $2.3 \pm 1.6$ & $2.0 \pm 1.7$ & $3.3 \pm 1.0$ & $P=0.096$ \\
\hline \multirow[t]{2}{*}{ XEN } & $1.1 \pm 1.4$ & $1.2 \pm 1.4$ & $0.7 \pm 1.2$ & $P=0.521$ \\
\hline & $P=0.025^{*}$ & $P=0.006^{*}$ & $P=0.051$ & \\
\hline
\end{tabular}


and in the OR $(P=0.84$, Table 1$)$. In-clinic needling tended to be more successful compared to in-OR needling ( $28 \%$ vs. $20 \%$ in the OR), although this was not statistically significant at any time point $(P=0.27$ at 6 months; Table 2). Kaplan-Meier survival analysis with $\log$ rank test showed that there was no difference in survival of success between clinic vs. the OR $(P=0.82)$. There were also no differences in repeat needling success rate (Supplemental Table 1), mean IOP and number of medications (Fig. 1, Fig. 2, Supplemental Table 2), and complication rate $(0 \%$ vs. $7 \%, P=0.32)$ between needling performed in clinic vs. the OR within 6 months. There was no difference in bleb needling patients requiring an additional IOP lowering procedure within 6 months $(15.6 \%$ vs. $26.7 \%, P=0.37$, Table 3$)$.

\section{Trabeculectomy vs. ExPress shunt vs. XEN gel stent}

Fourteen patients $(29.8 \%)$ had trabeculectomy, 16 patients (34.0\%) had ExPress shunt, and 17 patients (36.2\%) had XEN gel stent. The average time from initial surgery to bleb needling ranged from 15 days to 12 years. However, the time to needling was significantly less $(P<$ $0.001)$ for XEN gel stent (1.7 \pm 1.7 months) compared to trabeculectomy (77.4 \pm 48.1 months) and ExPress shunt (39.4 \pm 24.7 months). XEN gel stent patients were also on significantly fewer medications prior to undergoing needling $(P=0.03$, Table 1$)$. There was no statistically significant difference in success rate between bleb needling of trabeculectomy, ExPress shunt, or XEN gel stent procedures at any time point $(29 \%$ vs. $38 \%$ vs. $12 \%$ at 6 months, $P=0.23$; Table 2 and Fig. 3). Kaplan-Meier survival analysis with log rank test also showed that there was no difference in survival of success between trabeculectomy vs. ExPress vs. XEN $(P=0.23)$. There were no differences in mean IOP or mean number of medications between the three procedures at any time point (Supplemental Table 2).

\section{Multinomial logistic regression predicting 6 month success}

A multinomial logistic regression of 6 month bleb needling success including bleb needling location, initial procedure type, baseline IOP, and baseline number of glaucoma medications showed no variables were significantly associated with an increase or decrease in the odds ratio of success (Supplemental Table 3).

\section{Re-needling rate}

The re-needling rate was defined as the number of patients that required needling procedures performed within 3 months of the most recent needling, and it was calculated for each group (Supplemental Table 4). Overall, $25 \%$ of clinic and $7 \%$ of the OR needling procedures were repeat needling procedures $(P=0.136)$. The reneedling rate was 22,13 , and $18 \%$ for trabeculectomy, ExPress shunt, and XEN gel stent, respectively $(P=0.92)$.

\section{Complications}

Only one bleb needling patient had a vision-threatening complication. This 81-year-old female patient had a kissing suprachoroidal hemorrhage 4 days after a bleb needling in the OR after failed trabeculectomy and required urgent drainage. Two months after drainage, her vision improved to 2 lines less than her preoperative best corrected VA due to development of chronic cystoid macular edema and epiretinal membrane. At this time point IOP was $7 \mathrm{mmHg}$ on 0 medications. There were no other vision-threatening complications including endophthalmitis, hypotony, subconjunctival hemorrhage, shallow anterior chamber, peripheral choroidal detachments, or other complication that led to a vision loss greater than 2 Snellen lines.

\section{Discussion}

Our study compared the success (defined as IOP $\leq 18$ $\mathrm{mmHg}$ on 0 glaucoma medications and without requiring an additional IOP lowering procedure within 6 months) after bleb needling performed in the clinic vs. the OR for prior trabeculectomy, ExPress shunt, or XEN gel stent. Success rate, mean IOP, and mean number of medications was similar in patients who received needling in clinic vs. the OR $(P>0.05)$. These results suggest that outcomes after bleb needling in the clinic vs. the OR are equivalent, and they highlight the lack of benefit

Table 2 Bleb revision success rate at each time point

\begin{tabular}{lllll}
\hline & $\mathbf{1 w}$ & $\mathbf{1} \mathbf{m}$ & $\mathbf{2} \mathbf{m}$ & $\mathbf{6} \mathbf{m}$ \\
\hline Clinic & $43.8 \%(14 / 32)$ & $31.3 \%(10 / 32)$ & $31.3 \%(10 / 32)$ & $28.1 \%(9 / 32)$ \\
OR & $53.3 \%(8 / 15)$ & $40 \%(6 / 15)$ & $20 \%(3 / 15)$ & $20 \%(3 / 15)$ \\
$P$ value & 0.539 & 0.555 & 0.422 & 0.552 \\
Trabeculectomy & $64.3 \%(9 / 14)$ & $50 \%(7 / 14)$ & $28.6 \%(4 / 14)$ & $28.6 \%(4 / 14)$ \\
EXPress & $43.8 \%(7 / 16)$ & $37.5 \%(6 / 16)$ & $37.5 \%(6 / 16)$ & $17.5 \%(6 / 16)$ \\
XEN & $35.3 \%(6 / 17)$ & $17.6 \%(3 / 17)$ & $17.6 \%(3 / 17)$ & 0.227 \\
P value & 0.261 & 0.157 & 0.442 & \\
\hline
\end{tabular}

Abbreviations: $w$ week, $m$ month(s) 
to performing a more costly version of the same procedure in the OR. Bleb needling performed in the clinic has a number of desirable advantages for patients, including decreased health care costs and a faster procedure time. Surgeons would also have the opportunity to treat a fibrotic bleb immediately in clinic rather than spending time and healthcare resources to schedule a patient in the OR. However, increased patient comfort with sedation in the OR is a key benefit that may influence patient-physician decision making. Our study found an overall success rate for bleb needling to be $25.5 \%$ at 6 months $(28.1 \%$ in clinic vs. $20 \%$ in the OR). Thus, bleb needling may limit the necessity for further treatment with glaucoma drops or additional IOP lowering surgery in some patients. This makes it a worthwhile surgery salvaging technique, especially when done in clinic. The theoretical advantage of performing bleb needling in the OR would be a safer and more controlled environment. However, in our study, vision-threatening complications were rare, and it only occurred in one patient who received needling in the OR. This patient had a history of



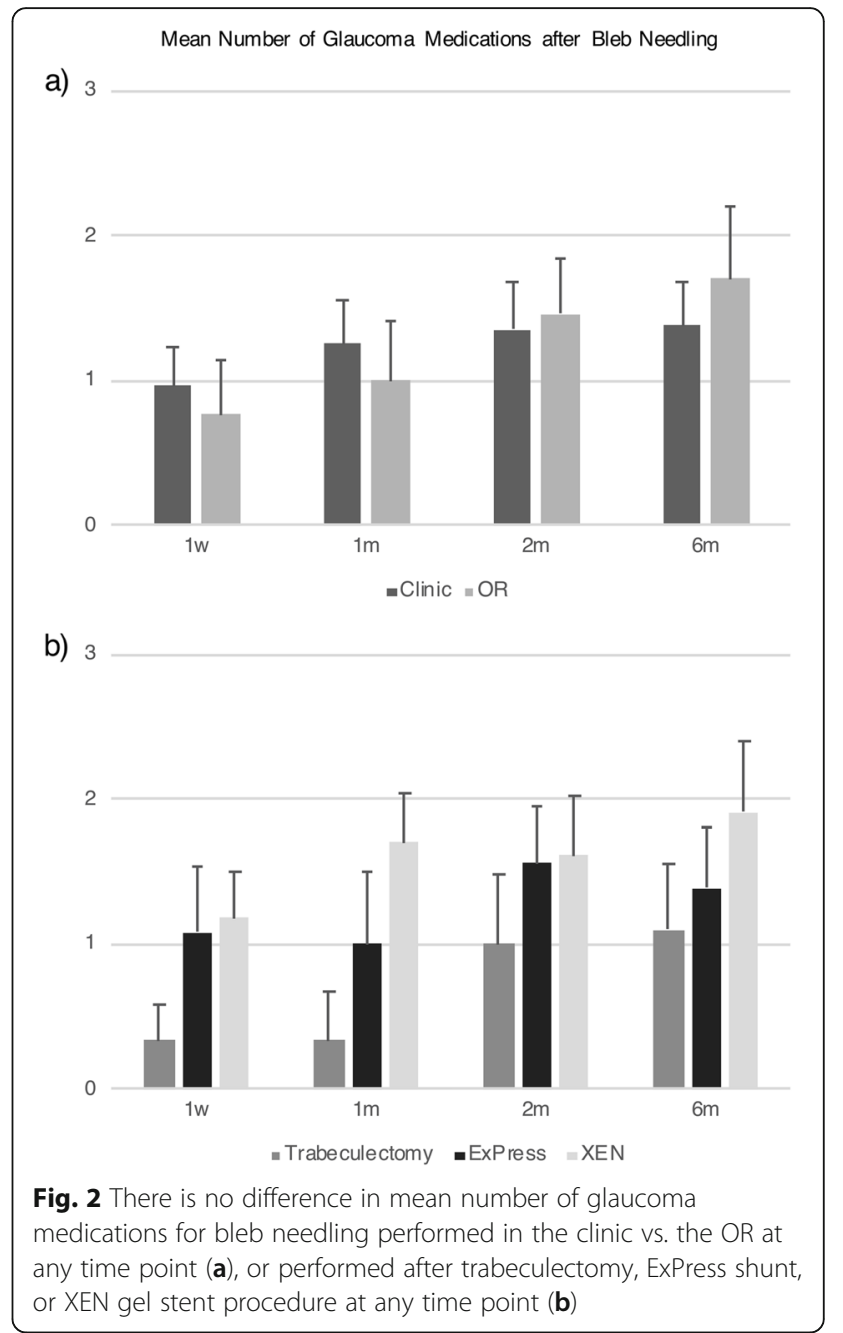

successful bleb needling of a failed trabeculectomy in clinic in the fellow eye, and from a baseline IOP of 19 $\mathrm{mmHg}$ on 4 classes of medications she achieved 10 $\mathrm{mmHg}$ off all medications postoperatively. The patient however preferred needling of the other eye in the OR under sedation to reduce discomfort. The procedure was unremarkable, and the IOP was successfully lowered from $21 \mathrm{mmHg}$ on 4 classes to $4 \mathrm{mmHg}$ off all

Table 3 Additional Procedure Rate ${ }^{a}$

\begin{tabular}{ll}
\hline Clinic & $15.6 \%(5 / 32)$ \\
OR & $26.7 \%(4 / 15)$ \\
$P$ value & 0.370 \\
Trabeculectomy & $42.9 \%(6 / 14)$ \\
EXPress & $25.0 \%(4 / 16)$ \\
XEN & $29.4 \%(5 / 17)$ \\
$P$ value & 0.746
\end{tabular}

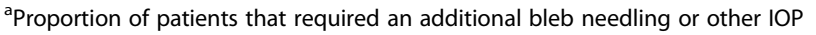
lowering procedure (cataract extraction, MicroPulse transscleral cytophotocoagulation, Ahmed valve, or Kahook dual blade goniotomy) within 6 months 

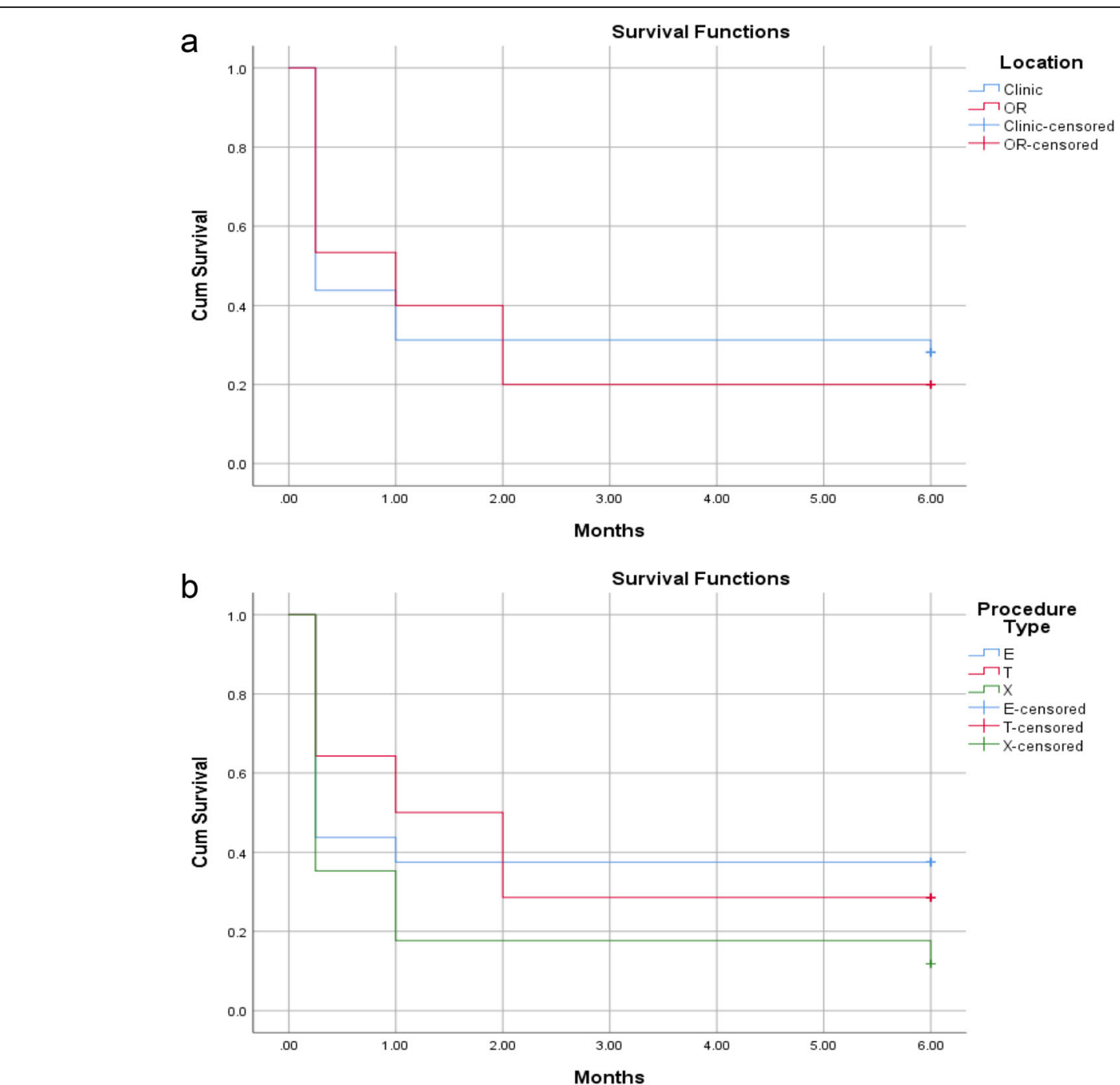

Fig. 3 There is no difference in Kaplan-Meier survival of success for bleb needling performed in the clinic vs. the OR $(P=0.82$, a) or after trabeculectomy vs. ExPress shunt vs. XEN gel stent $(P=0.23, \mathbf{b})$

medications on postoperative day 1 . The patient returned back to emergency clinic on day 4 with sudden loss of vision in this eye, and she was found to have kissing suprachoroidal hemorrhage requiring urgent drainage. Furthermore, the only difference in technique between needling performed in the clinic vs. the OR was releasing posterior adhesions of the bleb in the OR. Our study suggests that this extra step did not yield any improvement in success. Releasing posterior adhesions has a higher risk of injuring posterior blood vessels that have a high tendency to bleed and may increase the risk of fibrosis, so we would recommend not pursuing posterior adhesions based off the results of our study.

Secondary analysis revealed no difference of needling outcomes in patients with prior trabeculectomy, ExPress shunt, and XEN gel stent. However, baseline characteristics were different. Baseline IOP was similar between the procedure groups, but patients with XEN gel stent were on significantly fewer medications prior to bleb needling $(1.1 \pm 1.3, P=0.03)$. The average time from the surgery to bleb needling was also significantly shorter with XEN $(1.7 \pm 1.7$ months, $P<0.001)$. This may be explained by the fact that XEN gel stent is a newer procedure and reflects the authors' beliefs that needling after XEN may be more effective when performed early in the postoperative period before adding back multiple medications. This also explains the higher than average bleb needling rate for XEN gel stent in this study (53.1\% in this study vs. up to $44.1 \%$ in the existing literature $[4,10-12])$. This is in contrast to patients with failed trabeculectomy or ExPress shunt who are more likely be treated with maximum tolerable medications, often by outside providers, before proceeding with bleb needling given concerns for higher risk of hypotony and vision-threatening 
complications [13]. Furthermore, bleb needling of XEN gel stent on average had led to equivalent final IOP and number of medications compared to bleb needling of trabeculectomy and ExPress shunt (Supplemental Table 2 ). The results of the multinomial logistic regression show XEN gel stent has a high odds (6.09) of success when baseline IOP, baseline medications, and needling location are controlled for compared to trabeculectomy. Though this finding was not statistically significant, a difference may be appreciated with a larger sample size.

There were nine patients in our study that had repeat bleb needling ( 5 in clinic and 4 in the OR) within 3 months. Of these patients only one who had a repeat needling of XEN gel stent in the clinic was able to maintain a successful outcome at the 6-month time point. Five of nine repeated bleb needling procedures were failures at 1 week follow up, and they received alternative interventions to lower IOP. Three patients that had initially received bleb needling in the clinic were repeated in the OR, and all of these cases failed by 1 month following the OR needling. These findings suggest that repeating bleb needling may not be efficacious and repeating failed clinic bleb needling in the OR may not improve outcomes.

The limitations of our study are the retrospective nonrandomized design and small sample size that limited subgroup analysis. Furthermore, there may be sampling bias from the individual circumstances of each patient that led the physician to choose bleb needling in the clinic vs. the OR. Also, the population of this sample was $85 \%$ Caucasian, which does not reflect the typical mixture of patients seen by an average ophthalmologist. However, to the best of our knowledge, this is the first study that analyzed various outcome measures after bleb needling performed in the clinic vs. the OR.

\section{Conclusions}

Our study shows that bleb needling with MMC can be effectively and safely performed in the clinic following failed trabeculectomy, ExPress shunt, or XEN gel stent. Future studies will involve a larger sample size and a prospective randomized study design comparing predictive factors and outcomes of needling after various blebforming procedures to better elucidate the best way to manage a failed bleb, an often unavoidable outcome of any bleb-forming procedure.

\section{Supplementary Information}

The online version contains supplementary material available at https://doi. org/10.1186/s12886-021-01870-1.

Additional file 1: Table S1. Bleb revision success rate after $>1$ needling procedures at each time point.

Additional file 2: Table S2. Mean IOP and number of medications.
Additional file 3: Table S3. Multinomial logistic regression for 6 month success.

Additional file 4: Table S4: Reneedling rate.

Additional file 5.

Abbreviations

OR: Operating room; MMC: Mitomycin C; IOP: Intraocular pressure; VA: visual acuity

Acknowledgements

Not applicable.

Authors' contributions

VP collected data, analyzed data, and composed manuscript. VN aided in data analysis and manuscript composition. JAA provided critical insight for interpretation of results and writing the manuscript. All authors read and approved the final manuscript.

Funding

The authors declare that they have received no funding for this study.

Availability of data and materials

All data generated or analyzed during this study are included in this published article [and its supplementary information files].

Ethics approval and consent to participate

All procedures performed in studies involving human participants were in accordance with the ethical standards of the institutional and/or national research committee and with the 1964 Helsinki declaration and its later amendments or comparable ethical standards. The need for consent was waived by the University of Missouri IRB (\#00000731).

Consent for publication

Not applicable.

Competing interests

The authors declare that they have no competing interests.

Received: 20 April 2020 Accepted: 16 February 2021

Published online: 04 March 2021

\section{References}

1. Lin S. Building a safer trabeculectomy. Br J Ophthalmol. 2006;90:4-5

2. Cairns JE. Trabeculectomy. Preliminary report of a new method. Am J Ophthalmol. 1968:66:673-9.

3. Sarkisian SR. The ex-press mini glaucoma shunt: technique and experience. Middle East Afr J Ophthalmol. 2009;16:134-7.

4. De Gregorio A, Pedrotti E, Stevan G, Bertoncello A, Morselli S. XEN glaucoma treatment system in the management of refractory glaucomas: a short review on trial data and potential role in clinical practice. Clin Ophthalmol Auckl NZ. 2018;12:773-82.

5. Ewing RH, Stamper RL. Needle revision with and without 5-fluorouracil for the treatment of failed filtering blebs. Am J Ophthalmol. 1990;110:254-9.

6. Shetty RK, Wartluft L, Moster MR. Slit-lamp needle revision of failed filtering blebs using high-dose mitomycin C. J Glaucoma. 2005;14:52-6.

7. Midha N, Gillmann K, Chaudhary A, Mermoud A, Mansouri K. Efficacy of needling revision after XEN gel stent implantation: a prospective study. J Glaucoma. 2020:29:11-4

8. Allan EJ, Jones JM, Ding K, Khaimi MA. Outcomes of bleb revision with mitomycin C after ex-PRESS shunt surgery. J Glaucoma. 2016;25:e318-23.

9. Lin S, Byles D, Smith M. Long-term outcome of mitomycin C-augmented needle revision of trabeculectomy blebs for late trabeculectomy failure. Eye (Lond). 2018:32:1893-9.

10. Arnljots TS, Kasina R, Bykov VJN, Economou MA. Needling with 5fluorouracil (5-FU) after XEN gel stent implantation: 6-month outcomes. J Glaucoma. 2018;27:893-9.

11. Ibáñez-Muñoz A, Soto-Biforcos VS, Chacón-González M, Rúa-Galisteo O, Arrieta-Los Santos A, et al. One-year follow-up of the XEN ${ }^{\circledast}$ implant with mitomycin-C in pseudoexfoliative glaucoma patients. Eur J Ophthalmol. 2018;29(3):309. https://doi.org/10.1177/1120672118795063. 
12. Smith $M$, Charles R, Abdel-Hay A, Shah B, Byles D, et al. 1-year outcomes of the Xen45 glaucoma implant. Eye (Lond). 2019;33(5):761. https://doi.org/10.1 038/s41433-018-0310-1.

13. Broadway DC, Bloom PA, Bunce C, Thiagarajan M, Khaw PT. Needle revision of failing and failed trabeculectomy blebs with adjunctive 5-fluorouracil: survival analysis. Ophthalmology. 2004;111:665-73.

\section{Publisher's Note}

Springer Nature remains neutral with regard to jurisdictional claims in published maps and institutional affiliations.

Ready to submit your research? Choose BMC and benefit from:

- fast, convenient online submission

- thorough peer review by experienced researchers in your field

- rapid publication on acceptance

- support for research data, including large and complex data types

- gold Open Access which fosters wider collaboration and increased citations

- maximum visibility for your research: over $100 \mathrm{M}$ website views per year

At BMC, research is always in progress.

Learn more biomedcentral.com/submissions 